\title{
Predictors of Aspiration Pneumonia in the Elderly With Swallowing Dysfunction: Videofluoroscopic Swallowing Study
}

\author{
Joo Young Ko, $\mathrm{MD}^{1}$, Dae Youp Shin, $\mathrm{MD}^{1}$, Tae Uk Kim, MD, PhD'², Seo Young Kim, MD, $\mathrm{MS}^{1}$, \\ Jung Keun Hyun, $\mathrm{MD}, \mathrm{PhD}^{2,3}$, Seong Jae Lee, $\mathrm{MD}, \mathrm{PhD}^{2}$ \\ ${ }^{1}$ Department of Rehabilitation Medicine, Dankook University Hospital, Cheonan; \\ ${ }^{2}$ Department of Rehabilitation Medicine, Dankook University College of Medicine, Cheonan; \\ ${ }^{3}$ Department of Nanobiomedical Science and BK21 PLUS NBM Research Center for Regenerative Medicine, \\ Dankook University, Cheonan, Korea
}

Objective To identify the variables of videofluoroscopic swallowing study (VFSS) that are useful for predicting the risk of aspiration pneumonia in elderly patients with dysphagia.

Methods A total of 251 patients (aged 65 years or more) were included and divided into a pneumonia group $(\mathrm{n}=133)$ and a non-pneumonia group $(\mathrm{n}=118)$. The pneumonia group included patients who had been diagnosed with aspiration pneumonia, and individuals in the non-pneumonia group did not have pneumonia but were referred for VFSS. The medical records and results of VFSS were reviewed and compared between the groups retrospectively.

Results The pneumonia group exhibited a male preponderance and a higher 8-point Penetration-Aspiration Scale (8PPAS) score. The mean values of 8PPAS score for swallowing thick liquid and rice porridge was significantly higher in the pneumonia group. The pharyngeal delay time (PDT) and pharyngeal transit time (PTT) were significantly longer in the pneumonia group. The amounts of vallecular and pyriform sinus residue were increased in the pneumonia group. The delay in swallowing reflex and the decrease in laryngeal elevation were more frequently observed in the pneumonia group. Among those variables, PDT and PTT were identified as significant predictors of aspiration pneumonia based on logistic regression analysis.

Conclusion The present study delineated the findings of VFSS, suggesting an increased risk of aspiration pneumonia in elderly patients with dysphagia. The results demonstrate that prolonged PDT and PTT are significant predictors of aspiration pneumonia.

Keywords Aspiration pneumonia, Aged, Deglutition disorders, Fluoroscopy

Received April 4, 2020; Revised October 20, 2020; Accepted November 2, 2020; Published online April 14, 2021

Corresponding author: Seong Jae Lee

Department of Rehabilitation Medicine, Dankook University Hospital, 201 Mangyang-ro, Dongnam-gu, Cheonan 31116, Korea. Tel: +82-41-550-6641, Fax: +82-41-551-7062, E-mail: rmlee@dankook.ac.kr

ORCID: Joo Young Ko (https://orcid.org/0000-0003-0021-0377); Dae Youp Shin (https://orcid.org/0000-0003-1085-5507); Tae Uk Kim (https://orcid. org/0000-0001-9904-8781); Seo Young Kim (https://orcid.org/0000-0002-5351-8044); Jung Keun Hyun (https://orcid.org/0000-0001-9254-4424); Seong Jae Lee (https://orcid.org/0000-0001-7867-4695).

(c) This is an open-access article distributed under the terms of the Creative Commons Attribution Non-Commercial License (http://creativecommons.org/ licenses/by-nc/4.0) which permits unrestricted noncommercial use, distribution, and reproduction in any medium, provided the original work is properly cited. Copyright $\odot 2021$ by Korean Academy of Rehabilitation Medicine 


\section{INTRODUCTION}

Aspiration pneumonia is a type of lung infection that develops after the inhalation of a relatively large amount of bolus consisting of colonized oropharyngeal or upper gastrointestinal contents that exceed the immune system of the respiratory system [1]. Aspiration pneumonia is generally considered a part of community- and hospital-acquired pneumonias rather than a distinct disease entity [2]. The prevalence of aspiration pneumonia accounts for approximately $5 \%$ to $15 \%$ of cases of community-acquired pneumonia, but its proportion of hospital-acquired pneumonia is unknown $[3,4]$. Aspiration pneumonia increases the possibility of mechanical ventilation, the duration of stay in the intensive care unit (ICU), the length of hospital stay, and the mortality rate compared with non-aspiration pneumonia [5]. It has been reported that older age, male gender, lung disease, diabetes mellitus, severe dementia, and poor oral health are risk factors for aspiration pneumonia [6]. In the study of national trends in admission for aspiration pneumonia in the United States in 2002-2012, approximately $80 \%$ of patients with aspiration pneumonia were aged 65 years or older [7]. Furthermore, the length of hospital stay was longer, the overall total charge was higher and the mortality was increased in patients with aspiration pneumonia aged 65 years or older [7].

Dysphagia, which is a common disorder in the elderly [8], is also considered a risk factor for aspiration pneumonia $[6,9,10]$. It has been reported that approximately $15 \%-40 \%$ of the elderly population over 65 years is affected by dysphagia $[11,12]$, and $55 \%$ of elderly adults 70 years and older diagnosed with pneumonia presented with clinical signs of oropharyngeal dysphagia [13]. Only $20 \%$ of older patients with aspiration symptoms exhibit normal swallowing function in a videofluoroscopic swallowing study (VFSS), and laryngeal penetration is identified in approximately $40 \%$ and aspiration in $20 \%$ [8]. A previous study reported that oropharyngeal dysphagia is closely related to community-acquired pneumonia in elderly subjects regardless of functional status and comorbidities [9]. According to a report by the Agency for Healthcare Research and Quality (AHRQ), it is estimated that approximately one-third of patients with dysphagia develop pneumonia [14]. Therefore, appropriate evaluation of swallowing function, including VFSS, should be provided to all elderly patients with clinical features related to dysphagia and pneumonia [10].

However, variables of VFSS that are valuable in estimating the risk of aspiration pneumonia in the elderly with dysphagia are unknown. Only one study investigated the findings of VFSS in patients older than 65 years with aspiration pneumonia, but specific parameters were not analyzed or compared with elderly individuals without aspiration pneumonia [15]. The present study aims to identify the variables of VFSS that are useful for predicting the risk of aspiration pneumonia in elderly patients with dysphagia.

\section{MATERIALS AND METHODS}

\section{Subjects}

The medical records and results of VFSS of a total of 251 elderly patients (aged 65 years or older) who were referred for VFSS due to symptoms suggestive of dysphagia in the period between July 2013 and April 2020 were reviewed retrospectively. The patients were excluded if they had (1) acute or subacute brain injury or stroke within 1 year; (2) cranial neuropathy or other acute neurological disorders that could affect swallowing function; (3) prior surgery in the pharyngo-laryngeal area; or (4) anatomical or structural abnormalities in the head and neck. Among the subjects, 133 patients were diagnosed with aspiration pneumonia (pneumonia group), and 118 patients did not exhibit any evidence of pneumonia (non-pneumonia group). The diagnosis of aspiration pneumonia was based on a clinical presentation consistent with pneumonia associated with a history of witnessed aspiration or risk factors for aspiration, which include impaired consciousness, history of seizure, age, antipsychotic medications, enteral feeding when associated with gastric dysmotility, poor cough, altered mental status, gastroesophageal reflux, poor oral care, and chronic obstructive pulmonary disease $[2,3]$. The clinical presentation of pneumonia was defined as two or more of the following symptoms or signs: fever (temperature, $>38^{\circ} \mathrm{C}$ ), cough, sputum production, shortness of breath, pleuritic chest pain, crackles, or consolidation on examination of the chest together with an acute pulmonary infiltrate evident on chest radiographs compatible with pneumonia [5]. 


\section{VFSS}

The VFSS was performed following the protocol described by Logemann [16] with minor modifications. The video images were recorded with lateral projection and stored digitally at a speed of 30 frames per second while the patients swallowed 3-mL boluses of various consistencies mixed with contrast medium in the seated position. In the authors' dysphagia clinic, the order of the consistencies swallowed was three times swallowing of thick liquid (water-soluble barium sulfate diluted to $70 \%$ ), two times swallowing of rice porridge, two times swallowing of curd-type yogurt, and three times swallowing of thin liquid (water-soluble barium sulfate diluted to $35 \%$ ) followed by two times swallowing of drinking $5 \mathrm{~mL}$ of thin liquid from a cup. The VFSS was not performed if the patients could not cooperate or the medical condition of the patients was not stable enough for performing VFSS.

\section{Analyses of VFSS}

Video files of VFSS were reviewed and analyzed by a physiatrist who was experienced in VFSS reading without exposure to any clinical information. VFSS parameters associated with the swallowing process in the oral and pharyngeal phases, including the presence of penetration and aspiration, were analyzed. All the parameters of VFSS were defined according to Logemann's protocol as described in Table 1 [17-20]. The severity of airway invasion was measured by the final version of the 8-point Penetration-Aspiration Scale (8PPAS) [20]. Residues in valleculae and pyriform sinuses were defined as clearly visualized barium lining in the pharynx after swallowing and graded into 4 levels-level 1 (none); level 2 (minimal), $<10 \%$ of bolus; level 3 (moderate), from $10 \%$ to $50 \%$ of bolus; and level 4 (maximal), $50 \%$ of bolus [18]. Oral transit time (OTT) was measured as the time elapsed from the backward movement of the bolus until the bolus

Table 1. Definitions of VFSS parameters for evaluation of the swallowing process

\begin{tabular}{|c|c|}
\hline Parameter of swallowing process & Definition $^{\text {a) }}$ \\
\hline \multicolumn{2}{|l|}{ Oral phase } \\
\hline Lip closure & $\begin{array}{l}\text { Maintaining labial seal to ensure that no food or liquid falls from the } \\
\text { mouth }\end{array}$ \\
\hline Bolus formation & Mastication and preparation of a semicohesive or ball \\
\hline Oral transit time & $\begin{array}{l}\text { The time elapsed from the backward movement of the bolus until the } \\
\text { bolus head reached the lower edge of the mandible (normal, }<1.5 \\
\text { seconds) }\end{array}$ \\
\hline Residue of oral cavity & $\begin{array}{l}\text { Barium residue on the floor of the mouth, tongue, hard palate, and } \\
\text { anterolateral sulcus }\end{array}$ \\
\hline \multicolumn{2}{|l|}{ Pharyngeal phase } \\
\hline Triggering of pharyngeal swallow & $\begin{array}{l}\text { Elevation and anterior movement of hyoid and larynx with bolus head } \\
\text { passing the spot where the lower edge of the mandible crosses the } \\
\text { tongue base (normal, }<0.5 \text { seconds) }\end{array}$ \\
\hline Laryngeal elevation & $\begin{array}{l}\text { Getting closer and tilting forward of the arytenoids to the base of epi- } \\
\text { glottis and closing the airway as larynx elevates (normal, }>2 \mathrm{~cm} \text { ) }\end{array}$ \\
\hline Residue in the valleculae and pyriform sinus & Barium residue on vallecula and pyriform sinus after swallowing \\
\hline Pharyngeal delay time & $\begin{array}{l}\text { The time from the arrival of the bolus at the lower edge of the man- } \\
\text { dible until pharyngeal swallow was triggered }\end{array}$ \\
\hline Pharyngeal transit time & $\begin{array}{l}\text { The time from the arrival of the bolus head at the lower edge of the } \\
\text { mandible until the bolus tail passed through the cricopharyngeal re- } \\
\text { gion }\end{array}$ \\
\hline Presence of penetration & $\begin{array}{l}\text { Presence of the bolus within the laryngeal inlet but above the true vo- } \\
\text { cal cord }\end{array}$ \\
\hline Presence of aspiration & Passage of the bolus material beyond the level of the true vocal cord \\
\hline
\end{tabular}

VFSS, videofluoroscopic swallowing study.

${ }^{a)}$ Based on Logemann [16]. 
head reached the lower edge of the mandible [16]. Pharyngeal delay time (PDT) was measured as the time from the arrival of the bolus at the lower edge of the mandible until pharyngeal swallow was triggered [16]. Pharyngeal transit time (PTT) was measured as the time from the arrival of the bolus head at the lower edge of the mandible until the bolus tail passed through the cricopharyngeal region [16]. The longest time among the three swallowing of the thick liquid was selected for the time parameters. The vallecular and pyriform sinus residues were measured based on the largest amount of residue among all swallowing. Other abnormalities were judged to be present if they were observed at least once during the VFSS examination irrespective of consistency. The results were compared between the pneumonia and the non-pneumonia groups.

\section{Statistical analysis and study approval}

Statistical analyses were performed using SPSS for Windows version 25.0 (IBM, Armonk, NY, USA). Categorical parameters were compared by Pearson chi-square test, and numerical parameters between the groups were compared by the independent t-test with the exception of ordinal variables for which the Mann-Whitney U test was used. Logistic regression analysis was performed for the parameters that exhibited significant differences between the pneumonia and the non-pneumonia group. Significance was accepted for $\mathrm{p}$-values $<0.05$. The study protocol was approved by the Institutional Review Board of Dankook University Hospital (IRB No. 2020-07-005). The informed consent was waived.

Table 2. Baseline characteristics

\begin{tabular}{lccc}
\hline \multicolumn{1}{c}{ Characteristic } & Pneumonia group (n=133) & Non-pneumonia group (n=118) & p-value \\
\hline Sex & & & \\
\hline Male & $85(63.91)$ & $59(50.00)$ & $0.026^{\text {a) }}$ \\
\hline Female & $48(36.09)$ & $59(50.00)$ & 0.423 \\
\hline Age (yr) & $78.77 \pm 6.15$ & $78.06 \pm 7.63$ & 0.100 \\
\hline Intubation & $33(24.81)$ & $18(15.25)$ & $0.014^{\text {b) }}$ \\
\hline Duration of mechanical ventilation (day) & $14.36 \pm 18.34$ & $5.50 \pm 5.57$ & 0.101 \\
\hline ICU care & $63(47.37)$ & $41(34.75)$ & 0.252 \\
\hline Duration of ICU care (day) & $15.84 \pm 19.82$ & $11.07 \pm 21.79$ & $\left.0.040^{\mathrm{a}}\right)$ \\
Tracheostomy & $10(7.52)$ & $2(1.69)$ & $0.000^{\mathrm{a}}$ \\
\hline Inpatient & $132(99.24)$ & $92(77.97)$ & 0.372 \\
\hline Underlying disease & & $38(32.20)$ & 0.068 \\
\hline Diabetes mellitus & $50(37.59)$ & $62(52.54)$ & 0.692 \\
\hline Hypertension & $85(63.91)$ & $14(11.86)$ & 0.353 \\
\hline Chronic kidney disease & $18(13.53)$ & $20(16.95)$ & 0.994 \\
\hline Hip fracture & $17(12.78)$ & $24(20.34)$ & 0.368 \\
\hline Cancer & $27(20.30)$ & $39(33.05)$ & 0.436 \\
\hline Heart disease & $37(27.82)$ & $17(14.41)$ & 0.080 \\
\hline Lung disease & $24(18.05)$ & $21(17.80)$ & 0.170 \\
\hline Dementia & $36(27.07)$ & $11(9.32)$ & 0.434 \\
\hline Parkinson disease & $20(15.04)$ & $25(21.19)$ & 0.497 \\
\hline Old stroke & $23(17.29)$ & $2(1.69)$ & 0.064 \\
\hline Old traumatic brain injury & $4(3.01)$ & $3(2.54)$ & \\
\hline Spinal cord injury & $0(0)$ & & \\
\hline Vares & & & \\
\hline
\end{tabular}

Values are presented as number of patients (\%) or mean \pm standard deviation.

ICU, intensive care unit.

${ }^{\text {a)}}$ Pearson chi-square test, ${ }^{\text {b) }}$ independent t-test. 


\section{RESULTS}

The baseline characteristics of the subjects are present- ed in Table 2. More males were diagnosed with aspiration pneumonia $(\mathrm{p}=0.026)$. The mean duration between the onset of aspiration pneumonia and VFSS was $22.44 \pm 22.63$

Table 3. Comparison of the 8PPAS between groups

\begin{tabular}{lccc}
\hline & Pneumonia group $(\mathbf{n}=133)$ & Non-pneumonia group $(\mathbf{n}=118)$ & p-value \\
\hline Thick liquid & $3.10 \pm 2.83$ & $2.18 \pm 2.27$ & $0.004^{\mathrm{a})}$ \\
Rice porridge & $2.31 \pm 2.15$ & $1.80 \pm 1.79$ & $0.012^{\mathrm{a})}$ \\
Curd-type yogurt & $1.63 \pm 1.36$ & $1.46 \pm 1.17$ & 0.184 \\
Thin liquid & $2.54 \pm 2.40$ & $2.45 \pm 2.26$ & 0.774 \\
Drinking $5 \mathrm{~mL}$ of thin liquid from a cup & $2.44 \pm 2.04$ & $2.04 \pm 1.76$ & 0.125 \\
\hline
\end{tabular}

Values are presented as mean \pm standard deviation. 8PPAS, 8-point Penetration-Aspiration Scale.

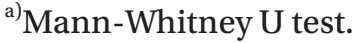

Table 4. Comparison of VFSS parameters

\begin{tabular}{|c|c|c|c|}
\hline & Pneumonia group $(n=133)$ & Non-pneumonia group $(\mathrm{n}=118)$ & p-value \\
\hline \multicolumn{4}{|l|}{ Time parameter } \\
\hline Oral transit time (s) & $5.97 \pm 10.74$ & $5.80 \pm 19.65$ & 0.933 \\
\hline Pharyngeal delay time (s) & $4.72 \pm 8.71$ & $2.48 \pm 4.00$ & $0.009^{\mathrm{a})}$ \\
\hline Pharyngeal transit time (s) & $5.33 \pm 8.73$ & $3.37 \pm 4.45$ & $0.025^{\mathrm{a})}$ \\
\hline \multicolumn{4}{|l|}{ Residue (grade) } \\
\hline Vallecular residue & $2.02 \pm 0.87$ & $1.76 \pm 0.86$ & $0.024^{\mathrm{b})}$ \\
\hline Pyriform sinus residue & $1.86 \pm 0.93$ & $1.53 \pm 0.90$ & $0.008^{\mathrm{b})}$ \\
\hline \multicolumn{4}{|l|}{ Other parameter } \\
\hline \multicolumn{4}{|l|}{ Lip closure } \\
\hline Complete & 122 & 113 & 0.192 \\
\hline Incomplete & 11 & 5 & \\
\hline \multicolumn{4}{|l|}{ Oral cavity residue } \\
\hline None & 66 & 71 & 0.094 \\
\hline Exist & 67 & 47 & \\
\hline \multicolumn{4}{|l|}{ Bolus formation } \\
\hline Adequate & 64 & 59 & 0.766 \\
\hline Inadequate & 69 & 59 & \\
\hline \multicolumn{4}{|c|}{ Triggering of pharyngeal swallowing } \\
\hline Normal & 28 & 38 & $0.045^{\mathrm{c})}$ \\
\hline Delayed & 105 & 80 & \\
\hline \multicolumn{4}{|l|}{ Laryngeal elevation } \\
\hline Intact & 38 & 52 & $0.011^{\mathrm{c})}$ \\
\hline Decreased & 95 & 66 & \\
\hline \multicolumn{4}{|l|}{ Pharyngeal wall coating } \\
\hline None & 19 & 26 & 0.110 \\
\hline Exist & 114 & 92 & \\
\hline
\end{tabular}

Values are presented as mean \pm standard deviation.

VFSS, videofluoroscopic swallowing study.

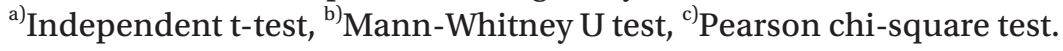


days. Most of the subjects underwent VFSS during hospitalization. The duration of mechanical ventilation was longer $(\mathrm{p}=0.040)$ and more tracheostomized patients ( $\mathrm{p}=0.014$ ) were noted in the pneumonia group.

The mean 8PPAS scores of each viscosity are presented in Table 3. For the thick liquid and rice porridge, the mean 8PPAS score was significantly increased in the pneumonia group compared with the non-pneumonia group ( $\mathrm{p}=0.004$ and $\mathrm{p}=0.012$, respectively).

The results of the VFSS parameters are presented in Table 4. For the time parameters, PDT and PTT were significantly prolonged in the pneumonia group compared with the non-pneumonia group $(\mathrm{p}=0.009$ and $\mathrm{p}=0.025$, respectively). The amounts of vallecular and pyriform sinus residue were greater in the pneumonia group compared with the non-pneumonia group $(\mathrm{p}=0.024$ and $\mathrm{p}=0.008$, respectively). The delay in swallowing reflex and the decrease in laryngeal elevation were more frequently observed in the pneumonia group ( $\mathrm{p}=0.045$ and $\mathrm{p}=0.011$, respectively).

To identify the significant variables for predicting the risk of aspiration pneumonia, logistic regression analysis was performed for the parameters that exhibited significant differences between the pneumonia and the non-pneumonia group. The results of logistic regression analysis showed that PDT (odds ratio $[\mathrm{OR}]=4.01 ; 95 \%$ confidence interval [CI], 1.267-12.703; $\mathrm{p}=0.018$ ) and PTT $(\mathrm{OR}=3.63 ;$ 95\% CI, 1.154-11.494; $\mathrm{p}=0.027$ ) were significant predictors of aspiration pneumonia (Table 5).

\section{DISCUSSION}

Swallowing dysfunction often results in aspiration of oral or gastric contents, which can cause aspiration pneumonia [2]. Given its pathophysiologic characteristics, swallowing dysfunction is associated with various risk factors [7]. In a previous systematic review, age, male gender, dysphagia, dementia, angiotensin I-converting

Table 5. Logistic regression analysis for predicting aspiration pneumonia

\begin{tabular}{|c|c|c|c|}
\hline Variable & OR & 95\% CI & p-value \\
\hline Sex, male & 1.61 & $0.817-3.182$ & 0.168 \\
\hline Duration of intubation & 1.05 & $0.953-1.146$ & 0.351 \\
\hline Tracheostomy & 5.49 & $0.398-75.690$ & 0.203 \\
\hline \multicolumn{4}{|l|}{ Thick liquid } \\
\hline Penetration (PAS 2-5) & 1.13 & $0.492-2.598$ & 0.772 \\
\hline Aspiration (PAS 6-8) & 2.57 & $0.272-24.323$ & 0.410 \\
\hline \multicolumn{4}{|l|}{ Rice porridge } \\
\hline Penetration (PAS 2-5) & 1.64 & $0.698-3.869$ & 0.256 \\
\hline Aspiration (PAS 6-8) & 0.52 & $0.119-2.309$ & 0.393 \\
\hline \multicolumn{4}{|l|}{ VFSS parameters } \\
\hline Pharyngeal delay time & 4.01 & $1.267-12.703$ & $0.018^{*}$ \\
\hline Pharyngeal transit time & 3.63 & $1.154-11.494$ & $0.027^{*}$ \\
\hline \multicolumn{4}{|l|}{ Vallecular residue } \\
\hline Minimal & 0.81 & $0.123-5.263$ & 0.821 \\
\hline Moderate & 0.49 & $0.068-3.544$ & 0.480 \\
\hline Maximal & 1.18 & $0.138-10.24$ & 0.882 \\
\hline \multicolumn{4}{|l|}{ Pyriform sinus residue } \\
\hline Minimal & 2.68 & $0.540-13.275$ & 0.228 \\
\hline Moderate & 2.36 & $0.389-14.255$ & 0.351 \\
\hline Maximal & 3.91 & $0.536-28.540$ & 0.179 \\
\hline Delayed triggering of pharyngeal swallowing & 1.48 & $0.663-3.291$ & 0.339 \\
\hline Decreased laryngeal elevation & 1.39 & $0.679-2.825$ & 0.370 \\
\hline
\end{tabular}

OR, odds ratio; CI, confidence interval; PAS, Penetration-Aspiration Scale; VFSS, videofluoroscopic swallowing study. ${ }^{*} \mathrm{p}<0.05$. 
enzyme deletion genotype and use of angiotensin-converting enzyme inhibitors were identified as risk factors for aspiration pneumonia [6]. However, no difference was observed in the present study with the exception of the male preponderance in the pneumonia group. The difference was not observed likely because the pneumonia group was compared with patients with dysphagia symptoms not with the normal subjects.

The pneumonia group in the present study exhibited higher 8PASS with high viscosity boluses, such as thick liquid and rice porridge ( $\mathrm{p}=0.004$ and $\mathrm{p}=0.012$, respectively). Previous studies have reported that swallowing function can be improved by increasing bolus viscosity in neurological patients and that the incidence of penetration and aspiration can be reduced [21,22]; however, the amounts of oral and/or pharyngeal residue and the risk of post-swallow airway invasion may also be increased [23]. The results of the present study suggest that a highviscosity diet may not decrease the risk of aspiration pneumonia in patients with dysphagia. However, generalization of such speculation is limited because the control group in the present study consisted of subjects with symptoms of dysphagia that were not essentially normal.

In the present study, the PDT, representing the time interval for initiation of the swallowing reflex, was significantly delayed in the pneumonia group and revealed as a significant predictor. Logemann [16] stated that pharyngeal swallowing is initiated when laryngeal elevation begins. Kim et al. [24] emphasized the importance of appropriate timing of the hyolaryngeal elevation for the following reasons: (1) epiglottic closure can prevent premature bolus leakage of material into the laryngeal vestibule before initiation of pharyngeal swallowing and (2) continuous bolus movement through the pharynx into the esophagus can be accomplished through opening of the upper esophageal sphincter in a proper timing. Following this description, a longer pharyngeal delay time may indicate an increased risk of laryngeal penetration or aspiration.

The present study showed that PTT was also significantly delayed in the pneumonia group and another significant predictor. Previous studies demonstrated that prolonged PTT can increase the incidence of aspiration pneumonia in some patient groups, including stroke or Parkinsonism [25-28]. It seems that delayed PTT can increase the risk of aspiration pneumonia in the general elderly group as well as specific patient groups, such as stroke or Parkinsonism. In a previous case-control study, Almirall et al. [9] demonstrated that $91.7 \%$ of elderly patients with community-acquired pneumonia have oropharyngeal dysphagia using the volume-viscosity swallow test. In another study by Park et al. [15], 70 of 105 patients with aspiration pneumonia exhibited abnormal findings during the oral or pharyngeal phase or both in VFSS. The present study provided stronger evidence that oropharyngeal dysphagia can be more severe in patients with aspiration pneumonia using quantitative analysis for time parameters not attempted in previous studies.

The time parameters, such as OTT, PDT, and PTT, were prolonged in the non-pneumonia group as well as the pneumonia group, when referring to the normal range generally accepted (OTT $<1.5$ seconds, PDT $<0.5$ second, PTT $<1.0$ second) [16]. Prolongation of those time parameters suggests that the subjects in the non-pneumonia group also had milder forms of swallowing dysfunction; however, they did not have aspiration pneumonia. However, increased prolongation of PDT and PTT indicates that the pneumonia group exhibits more advanced dysphagia.

The pneumonia group also exhibited decreased laryngeal elevation more frequently $(\mathrm{p}=0.011)$. In normal individuals, when the pharyngeal swallow is triggered, the larynx elevates and moves anteriorly to tuck itself under the base of the tongue as a component of airway protection [16]. During swallow, the larynx elevates approximately $2 \mathrm{~cm}$ in normal young adult men [29]. Reduced laryngeal elevation can result in inability of the arytenoids to tilt anteriorly enough to make good contact with the epiglottic base, leaving the entrance to the airways lightly open, allowing penetration of the bolus into the airway entrance, and this penetrated material is typically aspirated after the swallow [16]. The residues in the valleculae and pyriform sinuses were significantly more abundant in the pneumonia group $(\mathrm{p}=0.024$ and $\mathrm{p}=0.008$, respectively). The greater amount of food residue along with the longer stay in the pharynx may contribute to the increased chance of aspiration to the lung [16].

This study has some limitations. The control group with normal swallowing function could not be recruited and compared due to ethical issues. However, proving the significance of dysphagia in the development of aspiration pneumonia was possible by comparing the pneu- 
monia group with the non-pneumonia group with less severe dysphagia. Aspiration pneumonia was diagnosed by conventionally accepted criteria, but other causes of pneumonia could not be completely differentiated because the study was performed retrospectively. Patients with silent aspiration were included in the pneumonia group, but it is uncertain whether those patients were included in the non-pneumonia group. Discrepancy of patient configuration between groups may have affected the results. Although every effort was made to exclude secondary causes for dysphagia, a history of old neurological disorders could affect swallowing function. However, there was no significant difference in the proportion of underlying co-morbidities between the groups.

In conclusion, the present study delineated the findings of VFSS, suggesting an increased risk of aspiration pneumonia in elderly patients with dysphagia: delay of initiation of swallowing reflex, longer stay of bolus in the pharynx, greater amount of residue, and incomplete elevation of larynx. Increasing the viscosity of the diet may not be effective in reducing the risk of aspiration pneumonia in those patient group. Among the parameters, prolonged PDT and PTT were identified as significant predictors of aspiration pneumonia.

\section{CONFLICT OF INTEREST}

No potential conflicts of interest relevant to this article were reported.

\section{ACKNOWLEDGMENTS}

This research was supported by Basic Science Research Program through the National Research Foundation of Korea (NRF) funded by the Ministry of Education (No. 2018R1D1A3B07049300).

\section{AUTHOR CONTRIBUTION}

Conceptualization: Ko JY, Lee SJ. Methodology: Lee SJ, Ko JY, Sin DY. Formal analysis: Lee SJ, Ko JY, Hyun JK, Kim TU, Kim SY. Project administration: Lee SJ. Visualization: Lee SJ, Ko JY. Writing - original draft: Ko JY. Writing - review and editing: Lee SJ. Approval of final manuscript: all authors.

\section{REFERENCES}

1. Makhnevich A, Feldhamer KH, Kast CL, Sinvani L. Aspiration pneumonia in older adults. J Hosp Med 2019;14:429-35.

2. Mandell LA, Niederman MS. Aspiration pneumonia. N Engl J Med 2019;380:651-63.

3. Marik PE. Aspiration pneumonitis and aspiration pneumonia. N Engl J Med 2001;344:665-71.

4. Jeon I, Jung GP, Seo HG, Ryu JS, Han TR, Oh BM. Proportion of aspiration pneumonia cases among patients with community-acquired pneumonia: a singlecenter study in Korea. Ann Rehabil Med 2019;43:1218.

5. Reza Shariatzadeh M, Huang JQ, Marrie TJ. Differences in the features of aspiration pneumonia according to site of acquisition: community or continuing care facility. J Am Geriatr Soc 2006;54:296-302.

6. van der Maarel-Wierink CD, Vanobbergen JN, Bronkhorst EM, Schols JM, de Baat C. Risk factors for aspiration pneumonia in frail older people: a systematic literature review. J Am Med Dir Assoc 2011;12:344-54.

7. Wu CP, Chen YW, Wang MJ, Pinelis E. National trends in admission for aspiration pneumonia in the United States, 2002-2012. Ann Am Thorac Soc 2017;14:874-9.

8. Kocdor P, Siegel ER, Giese R, Tulunay-Ugur OE. Characteristics of dysphagia in older patients evaluated at a tertiary center. Laryngoscope 2015;125:400-5.

9. Almirall J, Rofes L, Serra-Prat M, Icart R, Palomera E, Arreola V, et al. Oropharyngeal dysphagia is a risk factor for community-acquired pneumonia in the elderly. Eur Respir J 2013;41:923-8.

10. Marik PE, Kaplan D. Aspiration pneumonia and dysphagia in the elderly. Chest 2003;124:328-36.

11. Barczi SR, Sullivan PA, Robbins J. How should dysphagia care of older adults differ? Establishing optimal practice patterns. Semin Speech Lang 2000;21:347-61.

12. Clave P, Shaker R. Dysphagia: current reality and scope of the problem. Nat Rev Gastroenterol Hepatol 2015;12:259-70.

13. Cabre M, Serra-Prat M, Palomera E, Almirall J, Pallares R, Clave P. Prevalence and prognostic implications of dysphagia in elderly patients with pneumonia. Age Ageing 2010;39:39-45.

14. Doggett DL, Tappe KA, Mitchell MD, Chapell R, Coates V, Turkelson CM. Prevention of pneumonia 
in elderly stroke patients by systematic diagnosis and treatment of dysphagia: an evidence-based comprehensive analysis of the literature. Dysphagia 2001;16:279-95.

15. Park S, Lee JY, Jung H, Koh SE, Lee IS, Yoo KH, et al. Use of videofluoroscopic swallowing study in patients with aspiration pneumonia. Ann Rehabil Med 2012;36:785-90.

16. Logemann JA. Evaluation and treatment of swallowing disorders. 2nd ed. Austin, TX: Pro-ED; 1998.

17. Han TR, Paik NJ, Park JW. The Functional Dysphagia Scale using videofluoroscopic swallowing study in stroke patients. J Korean Acad Rehabil Med 1999;23:1118-26.

18. Han TR, Paik NJ, Park JW. Quantifying swallowing function after stroke: a functional dysphagia scale based on videofluoroscopic studies. Arch Phys Med Rehabil 2001;82:677-82.

19. Paik NJ, Kim IS, Kim JH, Oh BM, Han TR. Clinical validity of the functional dysphagia scale based on videofluoroscopic swallowing study. J Korean Acad Rehabil Med 2005;29:43-9.

20. Rosenbek JC, Robbins JA, Roecker EB, Coyle JL, Wood JL. A penetration-aspiration scale. Dysphagia 1996;11:93-8.

21. Clave P, de Kraa M, Arreola V, Girvent M, Farre R, Palomera E, et al. The effect of bolus viscosity on swallowing function in neurogenic dysphagia. Aliment Pharmacol Ther 2006;24:1385-94.

22. Leonard RJ, White C, McKenzie S, Belafsky PC. Effects of bolus rheology on aspiration in patients with Dysphagia. J Acad Nutr Diet 2014;114:590-4.

23. Newman R, Vilardell N, Clave P, Speyer R. Effect of bolus viscosity on the safety and efficacy of swallowing and the kinematics of the swallow response in patients with oropharyngeal dysphagia: white paper by the European Society for Swallowing Disorders (ESSD). Dysphagia 2016;31:232-49.

24. Kim Y, McCullough GH, Asp CW. Temporal measurements of pharyngeal swallowing in normal populations. Dysphagia 2005;20:290-6.

25. Bisch EM, Logemann JA, Rademaker AW, Kahrilas PJ, Lazarus CL. Pharyngeal effects of bolus volume, viscosity, and temperature in patients with dysphagia resulting from neurologic impairment and in normal subjects. J Speech Hear Res 1994;37:1041-59.

26. Johnson ER, McKenzie SW, Rosenquist CJ, Lieberman JS, Sievers AE. Dysphagia following stroke: quantitative evaluation of pharyngeal transit times. Arch Phys Med Rehabil 1992;73:419-23.

27. Johnson ER, McKenzie SW, Sievers A. Aspiration pneumonia in stroke. Arch Phys Med Rehabil 1993;74:973-6.

28. Lin CW, Chang YC, Chen WS, Chang K, Chang HY, Wang TG. Prolonged swallowing time in dysphagic Parkinsonism patients with aspiration pneumonia. Arch Phys Med Rehabil 2012;93:2080-4.

29. Jacob P, Kahrilas PJ, Logemann JA, Shah V, Ha T. Upper esophageal sphincter opening and modulation during swallowing. Gastroenterology 1989;97:1469-78. 